\title{
Criterios \\ Proyecto w@ndA: hacia un entorno homogéneo de tramitación de expedientes en la Junta de Andalucía
}

Enrique Encina Encina

Coordinador del Proyecto w@ndA. D.G. Administración Electrónica y Calidad de los Servicios. Junta de Andalucia

\section{Resumen}

El Proyecto w@ndA nace del Plan Director de Organización para la Calidad de los Servicios (PLADOCS) de la Junta de Andalucia con el objetivo principal de ofrecer una visión homogénea a la ciudadanía de su relación con la Administración de la Junta de Andalucia. Para ello, el Proyecto w@ndA centra su actuación en cuatro líneas principales: identificar, racionalizar, simplificar, automatizar y poner a disposición de los ciudadanos todos los procedimientos de la administración que van a ser tramitados por aquellos canales que nos demanda la ciudadania; implantar las infraestructuras tecnológicas que sirvan de base a toda la tramitación de expedientes de forma automatizada; desarrollar las plataformas y los protocolos que nos permitan prestar servicios de atención a la ciudadania de una forma homogénea e independiente del canal utilizado; establecer nuevas normas e instar la modificación de las ya existentes, que sean necesarias para el establecimiento de los tres puntos anteriores.

\section{Palabras clave}

\section{Administración electrónica}

Andalucía

Expedientes

Junta de Andalucia

Proyecto W@anda

Servicios

\section{Punto de partida: la calidad al servicio de} la ciudadanía

La Administración de la Junta de Andalucía del siglo XXI se enfrenta a un cambio en el esquema de relación con el ciudadano: de un esquema de "Admistración-Ciudadano" se está evolucionando a uno de "Oferente-Demandante de servicios". Este esquema está caracterizado por un incremento en la exigencia de calidad de los servicios prestados y de incorporación de otros nuevos servicios, en muchas ocasiones derivados de la irrupción de las nuevas tecnologías; todo ello sin olvidar la eficacia y eficiencia en costes. Este fenómeno, que afecta de forma general a todas las Administraciones Públicas, se enmarca en una evolución más amplia en la Sociedad que, tras pasar de la era Industrial a la era de la información, a lo largo del siglo XX, se encuentra en plena transición desde dicha era de la Información a la Sociedad del Conocimiento.

En este escenario, las tecnologías de la información actúan como catalizador del proceso, constituyendo un elemento imprescindible para el funcionamiento de las organizaciones. El progreso y la garantía de supervivencia de las mismas están asociados a la correcta incorporación de los avances que se producen. La tecnología es el componente fundamental para el cambio y la innovación de procesos y servicios. De esta forma las aportaciones de las tecnologías de información y comunicaciones se pueden observar en diferentes ámbitos dentro de las administraciones públicas:

$>$ Incremento de la calidad del servicio

> Incorporación de nuevos servicios

$>$ Incremento de la calidad del trabajo y la satisfacción del empleado de la administración

$>$ Eficacia en los costes

Las administraciones públicas, en general, y la Junta de Andalucía, en particular, iniciaron su mecanización en la década de los 80, consolidando sus sistemas en los 90 . Tras aproximadamente veinte años de existencia se ha alcanzado un grado de madurez suficiente tanto de la organización como de los sistemas.

Es en esta situación donde se debe introducir un punto de inflexión importante que, partiendo de la adaptación al nuevo escenario de relaciones administración-ciudadanos (gráfico 1), sirva para conformar la Administración Andaluza del siglo XXI, caracterizada por :

> Nuevas formas de relaciones entre la administración y los ciudadanos.

> Adaptación de las funciones de los empleados, evolucionando hacia labores de asesoramiento y de mayor valor añadido.

> Incorporación de nuevos servicios acordes con las nuevas necesidades y requerimientos. 
$>$ Elevado nivel de calidad de los servicios prestados.

$>$ Una gestión eficaz de los costes de los servicios.

$>$ Y, en última instancia, la satisfacción del ciudadano.

\section{Antecedentes y oportunidad}

El desarrollo de iniciativas para la consecución de los objetivos previamente identificados se engloba bajo el epigrafe de e-Government o Administración Electrónica, ámbito de actuación preferente en las actuaciones que las administraciones públicas abordan para la consecución de la Sociedad del Conocimiento.

El Gartner's Group define la Administración electrónica o e-Government como: La innovación continua de los servicios públicos, la participación de los ciudadanos y la forma de gobernar mediante la transformación de las relaciones externas e internas a través de la tecnología, internet y los nuevos medios de comunicación.

El objetivo final de la Administración electrónica se podría describir como: "Todos los ciudadanos, sin ninguna excepción, acceden telemáticamente de forma habitual y natural a los servicios públicos".

El proceso de evolución hasta la plena Administración Electrónica sólo se desarrollará con la suficiente energía si se logra una más rápida introducción en el uso a nivel social de los medios tecnológicos y un incremento en la oferta de servicios en el área telemática.

Para ello, las administraciones públicas en el ámbito europeo, estatal y autonómico, dentro de sus distintos ámbitos de responsabilidad, han desarrollado una serie de planes de actuación estratégicos que impulsan acciones y proyectos tendentes a promocionar la utilización de medios digitales en los ámbitos en los que el sector público incide directamente.

En el ámbito de la Junta de Andalucía (gráfico 2) el avance hacia la administración electrónica ha tenido su reflejo en la acción de gobierno mediante dos instrumentos clave:

1. El Plan de Iniciativas Estratégicas para el Desarrollo de la Sociedad de la Información en Andalucía (i@landalus)

2. El Plan Director de Organización para la Calidad de los Servicios (PLADOCS)

Ambos responden a la misma filosofía y pretenden servir de motores para la más rápida introducción del tejido social y económico andaluz, por una parte, y de la Administración de la Comunidad Autónoma, por otra, en la Sociedad de la Información y el Conocimiento.

Estas iniciativas tienen como precedentes el Plan de Acción e-Europe 2002, aprobado por el Consejo Europeo en Feira, y el más cercano en el tiempo Plan de Acción e-Europe 2005 adoptado por el Consejo Europeo celebrado en Sevilla en junio de 2002.

El Plan Director de Organización para la Calidad de los Servicios, con una vigencia de 2002-2005, se configura en torno a cinco estrategias de carácter preferente, entre las que se encuentra la "Estrategia de Aplicación de las Tecnologías de la Información a la Administración en General".

Esta estrategia establece distintos programas que, por una parte, facilitan directamente los procedimientos que afectan a los ciudadanos y, por otra, sirven a una mejor utilización de los recursos humanos y materiales de la Junta de Andalucia que redunde en una mejora de la calidad de los servicios al disponer de mayores y más cualificados efectivos para realizar esta tarea. Uno de estos programas es el "Programa de Creación de un Sistema Integrado de Gestión de Expedientes".

\section{Arquitectura}

Los retos de la Administración Electrónica planteados en la Junta de Andalucía pasan en la actualidad por reducir las distancias con los ciudadanos, impulsar la difusión de los servicios existente y concebir la tecnología como instrumento de transformación de la gestión pública.

Estos retos exigen una adaptación de los sistemas de información que soportan los procesos realizados por la Junta de Andalucia.

Los objetivos generales en los que se basa la definición de dichos sistemas de información son:

> Accesibilidad: Administración transparente y abierta al ciudadano, aumentando el número de servicios ofrecidos a los mismos, mejorando la calidad de los mismos y reforzando el papel de terceros colaboradores en los procesos de gestión; es decir, establecer nuevas vías que faciliten el acceso del ciudadano a la Administración y a su catálogo de servicios. Además, esta apertura debe hacer transparente al ciudadano la estructura competencial de la Administración, convergiendo al concepto de Ventanilla Única.

> Conocimiento: consolidación de la información de forma óptima, para que pueda ser explotada y publicada a todos los niveles. La información así generada debe permitir el conocimiento de la realidad del entorno de un ciudadano, obtener información para la mejora de la gestión, análisis, simulación, etc., y, en definitiva, estructurar los datos y convertirlos en información, desarrollando así un modelo de conocimiento de la realidad de la Comunidad Andaluza.

> Eficacia: optimización de la actividad operativa de las administraciones, facilitando la agilidad y flexibilidad de la gestión y la racionalización de esfuerzos y recursos. El sistema resultante 


\section{Criterios}

Proyectow@ndA: hacia un entorno homogéneo de tramitación de expedientes en la Junta de Andalucía

\section{Enrique Encina Encina}

debe estar basado en un modelo único e integrado, en línea con las nuevas tendencias tecnológicas.

Estos tres objetivos generales han de apoyarse en un factor clave de éxito: la adaptación y orientación de las estructuras e inversiones en infraestructura tecnológica.

La Junta de Andalucia, para dar cobertura a los requerimientos derivados de la necesidad de implantar un modelo de Administración Electrónica corporativo, que potencie la accesibilidad del ciudadano a unos servicios públicos de calidad, pone en marcha el Proyecto.

El Proyecto w@ndA centra la atención sobre dos puntos de vista:

$>$ El del procedimiento administrativo (entendido como proceso), en la medida que implementa la regulación normativa que regula la prestación del servicio.

$>$ El de los sistemas de información por cuanto que soportan la mecanización de los procesos masivos y rutinarios y facilitan los intercambios de información y documentos.

A partir de lo expuesto, entendemos la Administración Electrónica como el conjunto de Procedimientos e Infraestructura Tecnológi-

Gráfico 1
ADMINISTRACIÓN
Mejora procesos internos
Acercamiento al ciudadano "Administración Abierta"
Agente tractor en la Sociedad de la Información
CIUDADANOS

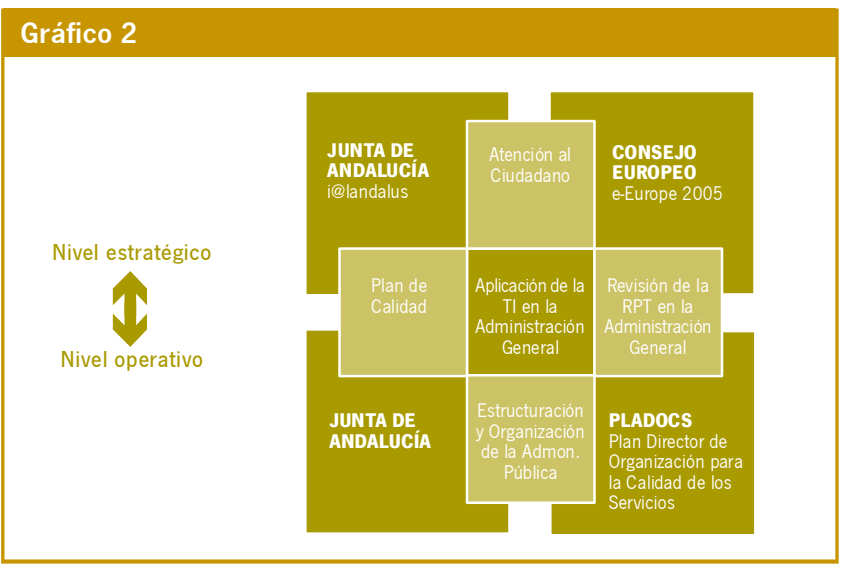

Gráfico 1. Los tres ejes de actuación de la Administración Pública con relación a las nuevas tecnologias de la información

ca soporte de los mismos que permiten el acceso telemático interno (gestores) y externo (ciudadanos) a los servicios ofrecidos por la Administración de la Junta de Andalucia, tanto para la consulta de información como para la teletramitación.

Adicionalmente el área de Procedimientos puede complementar los valores positivos que aportan los sistemas de información a través de aspectos como la normalización y simplificación de procedimientos. Con esto se trata de facilitar la relación del ciudadano con la administración cuando éste actúa como interesado en un procedimiento administrativo mediante la simplificación de la documentación a presentar, evitando duplicidades.

La interacción entre ambos ámbitos se potencia con un elemento integrador: Workflow. Este concepto permite combinar la racionalización y mejora de los procesos con los objetivos de negocio reflejados en los sistemas de información transaccionales.

\section{Procedimientos}

La administración de la Junta de Andalucia viene trabajando desde años atrás en la normalización, racionalización y simplificación de procedimientos. Una primea actuación sirvió para inventariar los procedimientos clasificándolo por tipologías para articular la normalización, simplificación y racionalización por grupos homogéneos.

Las actuaciones propuestas persiguen facilitar la relación del ciudadano con la Administración, a través de la simplificación de los procedimientos administrativos que le afectan. Este objetivo se puede concretar en otros más específicos:

> Reducir el número de registros mediante la unificación de los mismos.

> Simplificar la documentación a aportar por el ciudadano para la inscripción en los registros oficiales.

> Simplificar la gestión que deba realizar la administración para la tramitación de inscripción en los registros oficiales rediseñando los procedimientos.

$>$ Homogeneizar y adecuar los plazos de resolución y notificación de los procedimientos de inscripción en registros oficiales.

> Reducir en los posible los plazos de resolución y notificación de los procedimientos de inscripción en los registros oficiales.

> Continuar el proceso de simplificación extendiéndolo a otros grupos de procedimientos inventariado.

> Implantar el sistema de resolución inmediata. 
En primer lugar se precisa una labor de definición de una terminología común y de los conceptos que se van a manejar, este componente es lo que conocemos como Dominio semántico, incluyendo desde los conceptos más básicos hasta los más complejos incluyendo el formato universal de procedimiento que deberá ser adoptado por la Junta de Andalucía como modelo básico de referencia en la creación e intercambio de datos de tramitación en el ámbito de la Administración Autonómica.

Muy relacionado con la normalización de procedimientos es necesario abordar un trabajo de estandarización de todos los elementos presentes en los procedimientos de cara a su posterior apertura hacia los ciudadanos. Se trata de identificar, racionalizar, simplificar, automatizar y poner a disposición de los ciudadanos aquellos procedimientos que sean susceptibles de ser tramitados por medios telemáticos (fundamentalmente Internet). Esta labor se lleva a cabo mediante la elaboración de las Guías de Tramitación de Familias en primer lugar y la elaboración posterior de las Guías de Tramitación de Procedimientos.

En el ámbito del Proyecto w@ndA se define Familia de Procedimientos a la "agrupación no arbitraria de procedimientos bajo el criterio de similitud en el esquema básico de tramitación, documentación de entrada y salida e información". Cada Familia de Procedimientos contiene un esquema simplificado de todos los procedimientos que pertenecen a la familia, incluyendo su tramitación, documentación de entrada y de salida, información básica para la tramitación, información mínima que se debe proporcionar al ciudadano, e información mínima que se debe proporcionar a la dirección. Cada procedimiento que pertenezca a una Familia se generará a partir de la propia definición de la Familia añadiendo sus elementos particulares.

La elaboración de las Guias de Tramitación de Familias es el resultado de un análisis de todos los procedimientos en el ámbito de la Junta de Andalucía para orientarlos hacia el flujo de información superando la tradicional departamentalización de la gestión y para incorporar sistemas que incrementen la eficiencia en la gestión.

Las Guias de Tramitación para un procedimiento particular se originarán, por herencia, a partir de una estructura jerárquica de como máximo tres niveles. En definitiva supone caracterizar a cada procedimiento por un conjunto de datos, fases y transiciones de forma que procedimientos muy similares puedan ser fundidos en un único de más alto nivel, cuyas particularizaciones originarían los de bajo nivel. Con esto se pretende lograr una clasificación de los procedimientos dentro de un número reducido de Familias que a su vez podrían admitir una cierta especialización. El objetivo principal de estas guías es proporcionar al ciudadano una cuidada referencia en la tramitación del procedimiento, definir la información mínima que se va a proporcionar al ciudadano y a la dirección y adecuar la definición de cada procedimiento al contexto de la Administración Electrónica.
Otro aspecto de procedimiento importante son las metodologías de trabajo a seguir a la hora de construir el entorno de trabajo que marquen las directrices a seguir en el desarrollo de todos los proyectos derivados, facilite la coordinación entre ellos y garantice su integración una vez hayan sido finalizados.

Finalmente hay que considerar el soporte normativo con el que es necesario respaldar toda la solución. En este sentido cabe destacar de manera especial la firme disposición de convertir en regulación normativa aquellas Familias de Procedimientos que tengan especial relevancia en el ámbito de la tramitación de procedimientos en la Administración Andaluza.

\section{Infraestructura tecnológica}

Para los componentes tecnológicos que soporten la tramitación telemática se han identificado los siguientes requisitos:

> Adecuación a las infraestructuras de Administración Electrónica de la Junta de Andalucia: Ofrecer un conjunto de trámites que estén accesibles al ciudadano vía multicanal implica contar con una serie de plataformas tecnológicas que garantizan el procedimiento electrónico y con las que ya cuenta la Junta de Andalucía: Infraestructura de Clave Pública, Registro Telemático, Notario Electrónico y Notificaciones Telemáticas.

> Adaptación al modelo: Igualmente, las nuevas aplicaciones de tramitación o cualquier otro desarrollo que pudiera precisarse en los escenarios identificados deben respetar al máximo las especificaciones del Modelo de Tramitación que expone este documento.

> Coexistencia: Es necesario que todos los escenarios dejen abierta la posibilidad de respetar ciertas aplicaciones de tramitación actualmente disponibles, bien porque su sustitución sea tecnológicamente muy compleja o bien porque no se entienda necesaria u oportuna por cualquier otra causa; ello implica una coexistencia indefinida entre tales aplicaciones y cualquier desarrollo que se precise.

> Homogeneidad e Interoperatividad: Dado que el principal objetivo perseguido es alcanzar un entorno homogéneo de tramitación para cualquier procedimiento de la Junta de Andalucia, es precisa la interoperatividad de las soluciones propuestas con los tramitadores que sean respetados inicialmente.

Con estos requisitos, se dibuja el esquema conceptual de componentes tecnológicos reproducido en el gráfico 3. Como queda reflejado, la infraestructura tecnológica del Proyecto w@ndA se concibe como un framework (marco de trabajo) de tramitación en la Administración Andaluza compuesto por una serie de componentes que facilita tanto las bases teóricas como prácti- 


\section{Criterios}

Proyectow@ndA: hacia un entorno homogéneo de tramitación de expedientes en la Junta de Andalucía

\section{Enrique Encina Encina}

cas y que se engarzan entre sí para conseguir los objetivos de primer nivel expuestos al principio de este documento. Cada componente tiene definido unos objetivos particulares, unos requisitos (funcionales, legales, de integración y de estandarización), una ubicación, una arquitectura, etc, información que se recoge a continuación. Todos los componentes, a su vez, aparecen sustentados sobre los conceptos que ya se han introducido anteriormente: metodología, normativa, dominio semántico y guias de tramitación. Veamos a continuación cada uno de estos componentes.

\section{El Nodo de Información w@ndA (NIWA)}

En el plano más operativo, el Nodo de Información w@ndA (NIWA a partir de ahora) es el núcleo central del sistema. Se configura como el almacén central donde se recoge toda la información de tramitación de la Junta de Andalucía. Consiste fundamentalmente en una base de datos centralizada y estructurada en tres niveles: catálogo de procedimientos, información de tramitación e información a la dirección. Esta base de datos se alimentará principalmente de los motores de tramitación y de la herramienta de definición de procedimientos, generadores fundamentales de la información requerida; la información almacenada será reportada, principalmente, a las herramientas de presentación y fidelización, a los propios motores de tramitación y a las herramientas de gestión.

Las líneas de acción que deben orientar la definición del Nodo de Información w@ndA son:

$>$ Hacer acopio de información de tramitación de forma proactiva.

> Convertir el ingente volumen de datos disponible en información útil para el análisis: transformar, depurar, integrar, explotar, disponiendo de automatismos capaces de efectuar eficazmente estos procesos.

> Incorporar diferentes visiones de la información para dar servicio a las distintas necesidades de explotación: visión del ciudadano, del gestor interno, planificación de políticas y estrategias, etc.

> Desarrollar modelos de comportamiento, análisis y simulación.

\section{Herramientas de Tramitación Administrativa}

La información almacenada en el NIWA es generada, transformada, comunicada... en definitiva, tramitada, por los elementos de la plataforma que englobamos bajo el epígrafe de Herramientas de Tramitación Administrativa.

$>$ Definición de Procedimientos. Para permitir que el NIWA y los motores de tramitación sean alimentados por el personal responsable de los procedimientos de cada Consejería, no necesariamente con conocimientos técnicos, se hace necesario implementar una herramienta de diseño gráfico que permita la generación

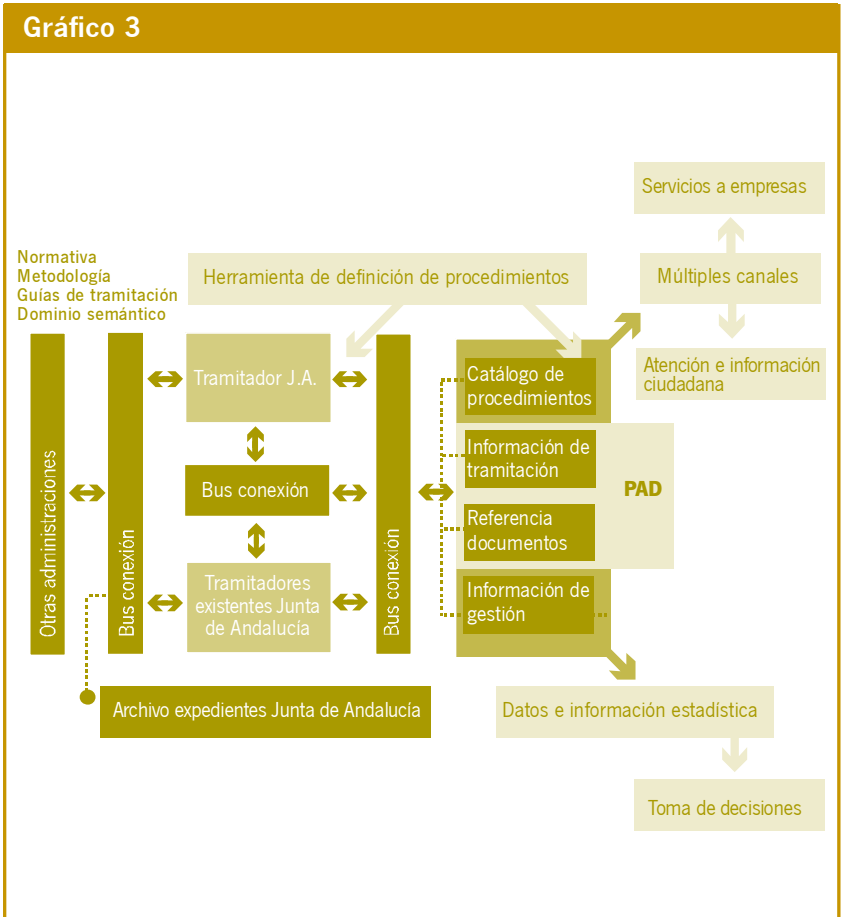

de procedimientos en ambos entornos de forma ágil a partir de requerimientos conceptuales-funcionales. En definitiva, será un traductor que, a partir de la definición del procedimiento realizada en un entorno gráfico, convierta dicha información en el código necesario para generar el procedimiento en el NIWA y en los motores de tramitación.

> Motor de Tramitación. Es la herramienta de base sobre la que se apoya la operativa de la Administración Electrónica. Definida en base a los estándares de la WfMC, permite la gestión homogénea de los procedimientos y alimenta coherentemente el NIWA.

$>$ Gestión Documental. Muy cercano al motor de tramitación se ubica este componente. Su funcionalidad en la plataforma w@ndA se concreta en ser el almacén de los documentos que participan en la tramitación, tanto los generados por el motor de tramitación durante la ejecución de los trámites como los documentos generados externamente y que se anexan a los trámites de los procedimientos, bien por el personal de la administración, bien por los ciudadanos en su relación con la administración.

> Presentación y Fidelización. Las líneas de acción en la definición del sistema de presentación y fidelización son tres: 1) desarrollar nuevos canales de interrelación orientados al ciudadano y al acceso del mismo a aplicaciones de servicio; 2) proporcionar acceso externo a los servicios desde una plataforma única; 3) reforzar y facilitar el papel de los ciudadanos en el proceso. 


\section{Herramientas de Intercambio de Información}

Estos componentes son de tipo instrumental y sirven para comunicar los elementos modulares del sistema entre sí de forma normalizada y segura. Este flujo de información debe reunir las condiciones necesarias para asegurar que se hace de una forma fiable. La fiabilidad de este trasiego de información implica, por una parte, asegurar que la información llegue a su destino; por otra, que sólo se comuniquen aquellos componentes que estén autorizados a hacerlo y en la forma que se haya preestablecido y, por último, que la información que llegue a su destino lo haga en el formato adecuado.

Con el objetivo de hacer estos requisitos lo más transparentes posibles a las herramientas que interactúan en estos procesos de flujo de información, se sitúa entre ellos una capa de abstracción que realice las funciones necesarias para hacer fiable la comunicación. Por tanto la misión de estas herrramientas de intercambio de información es recoger la información alli donde se produce (fundamentalmente de los motores de tramitación), verificar las primitivas sobre autorizaciones, realizar las transformaciones que sean necesarias y llevar los datos hasta el destinatario.

\section{Herramientas de Gestión}

De la misma forma que la explotación de la información del NIWA, de cara a los ciudadanos, pretende responder principalmente al ¿cómo va lo mío? la información hacia la Alta Dirección se orienta al ¿cómo puedo mejorar? La información almacenada en el NIWA debe permitir obtener los Indicadores Clave de Gestión que, cruzados según los diferentes ejes de agregación definidos y examinados con las correspondientes herramientas analiticas, respondan al gestor a las preguntas de qué se hace (nivel de servicio), cuándo y cómo se hace (eficiencia) y con qué recursos se hace (eficacia), entre otras.

Para que la información del NIWA llegue a la Alta Dirección con las características deseables de exactitud, agilidad, y sencillez es necesario implementar dos elementos de elaboración de dicha información: el componente de transformación y el de gestión propiamente dicho.

\section{Archivo Electrónico de Expedientes}

Los expedientes tramitados en la Junta de Andalucia, una vez cerrados, deben ser almacenados para su posterior consulta (interesados, tanto ciudadanos como administraciones, pero también historiadores, investigadores, etc.). Esta consulta debe asegurarse a futuro, considerando un horizonte a muy largo plazo, independientemente de las evoluciones tecnológicas que se produzcan.

Es por ello que el aspecto fundamental para la elaboración de este componente es la utilización de unos estándares de codificación de la información de los expedientes (datos y metadatos) y del sistema de almacenamiento y acceso.

\section{Organización}

La organización del proyecto debe necesariamente contar con elementos que faciliten la participación de todos los interesados con el nivel de compromiso necesario para garantizar el éxito del mismo. En este sentido se propone el modelo de coordinación representado en el gráfico 4.

El Comité de Seguimiento es el máximo órgano de coordinación del proyecto y tiene como funciones principales:

> Impulsar la formación de grupos y comisiones técnicas

> Impulsar la realización de los trabajos

$>$ Aprobar resultados

> Formalizar los resultados en el ámbito de la Administración de la Junta de Andalucía

El Comité de Seguimiento w@ndA, al tratarse de un proyecto directamente derivado de la "Estrategia de Aplicación de las Tecnologías de la Información en la Administración General" del Plan Director de Organización para la Calidad de los Servicios, se integra dentro de la Comisión de Seguimiento del Plan Director sin perjuicio de la puesta a disposición de todas sus actuaciones al Consejo Interdepartamental de Informática de la Junta de Andalucia y a la Comisión Interdepartamental para la Sociedad de la Información, al ser w@ndA, además, un proyecto de carácter horizontal que contribuye a la modernización de la Administración Andaluza, que tiene que ver con las aplicaciones y programas informáticos que se requieren para el funcionamiento del sistema regulado por el Decreto 183/2003 y que nace con el principal objetivo de mejorar la calidad de los servicios prestados al ciudadano a través del portal andaluciajunta.es.

En lo referente a la construcción de los componentes w@ndA es el "Grupo de Usuarios de Componentes" el órgano de coordinación. Este grupo está formado por representantes de todos los organismos de la Junta de Andalucia y tiene asignadas las siguientes funciones:

> Designar las Comisiones Técnicas

$>$ Definir requisitos y prioridades

$>$ Validar resultados

Este grupo de trabajo elige, de entre sus miembros, a un grupo más operativo, la "Comisión técnica de componentes", que tiene como misión:

> Preparar, adjudicar y supervisar la realización de los trabajos

$>$ Toma de decisiones

$>$ Elevar los resultados al Grupo de Usuarios

En materia de procedimientos es el "Grupo de Usuarios de Procedimientos" el máximo órgano de coordinación dentro del 


\section{Criterios}

Gráfico 4.Coordinación w@ndA

Proyectow@ndA: hacia un entorno homogéneo de tramitación de expedientes en la Junta de Andalucía

\section{Enrique Encina Encina}

\section{Gráfico 4}

\section{COMITÉ DE SEGUIMIENTO}

Grupo de Usuarios

Componentes w@ndA

Grupo de Usuarios/Catálogo de procedimientos

Comisión Técnica/Componentes w@ndA

Resp. Dominio Semántico, Metodologia y

Normativa

Resp. Componente de Definición y

Almacenamiento

Resp. Componente de Tramitación y

Documentación

Resp. Componente de Presentación y

Fidelización

Resp. Componente de Transformación y Gestión

Resp. Componentes de Conectividad

C.T. - Guías tramitación

Procedimientos Familia N

C.T. - Guías tramitación Familias C.T. - Guías tramitación Procedimientos Familia 1 C.T. - Guias tramitación Procedimientos Familia 2

\section{Gráfico 6}

\section{Proyecto}

Constitución Grupos de Usuarios

Constitución Comités Técnicos

Definición del Proyecto

Dominio Semántico

Metodología

Guías de Tramitación de Familias

Oficina Control de Calidad

Guias de Tramitación de Procedimientos

Componente de Definición

Componente de Tramitación

Componente de Almacenamiento

Componentes de Conectividad

Componente Documental

Componente de Presentación y Fidelización

Componente de Gestión

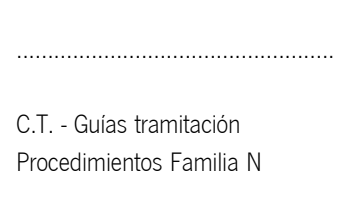

\section{Gráfico 7}

Sub-proyectos

1. Organización

2. Divulgación del proyecto

3. Constitución Comité Seguimiento

4. Constitución Grupos Usuarios

5. Constitución Comisiones

Técnicas

6. Definición

7. Definición Dominio Semántico

8. Guias de Tramitación de Familias

9. Guias Tramitación Procedimientos

10. Metodología

11. Normativa

12. Diseño Sistema de Gestión
Estado

Terminado

Terminado

Terminado

Terminado

Terminado

Terminado

En ejecución

En ejecución

En ejecución

En ejecución

En ejecución

En ejecución

Contratación

Pendiente
En ejecución
13. Desarrollo e implantación

14. Herramienta de Definición

15. Herramienta de Almacenamiento

16. Herramienta de Tramitación

17. Herramienta de Soporte Documental

18. Herramienta Presentación y Fidelización

19. Sistema de Gestión

20. Conectividad interna N1

21. Conectividad interna N2

22. Conectividad externa

23. Administración y gestión

24. Coordinación del Proyecto

25. Control de Calidad

\section{Gráfico 5}

Familias de tramitación de procedimientos

Familia 1 Procedimientos de autorizaciones, acreditaciones e inscripciones registrales

Familia 2 Procedimientos de subvenciones, becas y premios

Familia 3 Procedimientos de evaluación y certificación

Familia 4 Procedimientos de expropiación y reversión

Familia 5 Procedimientos de contratación pública

Familia 6 Procedimientos de sancionadores

Familia 7 Procedimientos de conciliación, mediación y arbitraje

Familia 8 Procedimientos de sugerencias, quejas y reclamaciones

Familia 9 Procedimientos de elaboración de disposiciones

Familia 10 Procedimientos de selección, contratación de personal y provisión de puestos

Familia 11 Procedimientos de prestaciones sociales y sanitarias

Familia 12 Procedimientos de responsabilidad patrimonial

Familia 13 Procedimientos de revisión de la actuación administrativa y reclamaciones previas

Familia 14 Procedimientos de convenios

Familias temáticas de procedimientos

Familia 15 Procedimientos de tesorería y deuda

Familia 16 Procedimientos tributarios y de otros ingresos de derecho público

Familia 17 Procedimientos de recursos humanos

Familia 18 Procedimientos de operaciones con bienes

Familia 19 Procedimientos sobre protección de menores

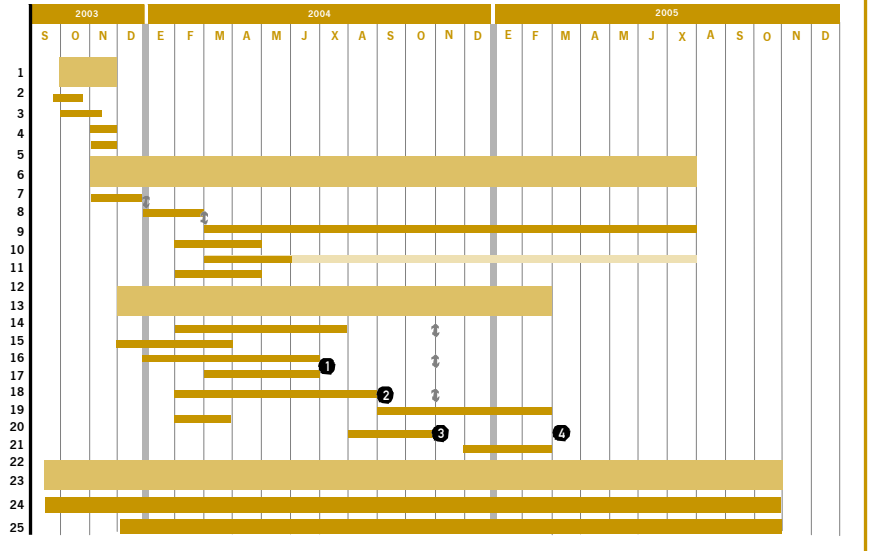


Proyecto w@ndA. Las funciones que desarrolla son análogas al "Grupo de Usuarios de Componentes".

Para el trabajo más operativo en este área, el Grupo de Usuarios de Procedimientos elige de entre sus miembros una "Comisión Técnica de Procedimientos" cuyas funciones son análogas a las de la "Comisión Técnica de Componentes".

\section{Plan de Acción}

El desarrollo de los componentes identificados a lo largo de este documento y, por lo tanto, la implementación de la solución w@ndA, permite identificar una serie de subproyectos que fueron planificados en la forma recogida en el gráfico 7.

\section{Situación actual}

A fecha de hoy, y en función de la planificación inicial, el Proyecto w@ndA presenta el aspecto del gráfico 6.

En cuanto a las Guías de Familias de Procedimientos, se han elaborado mediante la realización de 6 reuniones de seguimiento y 19 mesas de trabajo, con una media de 6 gestores por mesa, con un total de 104 participantes. El resultado son estas 19 Guías de Tramitación de Familias, 5 de ellas temáticas (gráfico 5).

En la actualidad se están realizando las Guias de Tramitación de Procedimientos comenzando con los procedimientos de la Familia 2: Procedimientos de Subvenciones, Becas y Premios. Para esta familia se está en proceso de elaboración de un decreto que regula la tramitación de todos los procedimientos de concesión de subvenciones en la Administración de la Junta de Andalucía.

También en el ámbito de la regulación normativa, se está elaborando la normativa que crea y regula el Nodo de Información w@ndA.

En cuanto a los componentes tecnológicos, se han adjudicado los contratos para la construcción de los siguientes:

Componente de Definición y Tramitación:Trew@y model@.

> Desarrollo a medida a partir de un motor de tramitación ya en funcionamiento en la Junta de Andalucía.

$>$ Código propiedad de la Junta de Andalucía

> 100\% Dominio Semántico de w@ndA

$>100 \%$ tecnología java (versión optimizada para SGBD Oracle)

$>100 \%$ integrado plataformas AE de la Junta de Andalucía

$>$ Modelo de componentes similar a la WfMC

$>$ Funcionalidad incorporada
> Model@: herramienta gráfica de modelado (XPDL)

$>$ Portafirmas electrónico

> Gestión de "avisos"

$>$ Escritorio de tramitación

Nodo de Información (Niw@)

$>$ Desarrollo sobre las estructuras que conforman el repositorio del conjunto de herramientas de Siebel (mucha funcionalidad ya viene desarrollada)

> Filosofía 100\% CRM

$>100 \%$ tecnología java (soporte linux en enero 2006)

$>$ Acceso ilimitado desde los SS.II. de la Junta de Andalucía a través del Bus de Conexión N1

> Explotación de la Información a través del Componente de Presentación y Fidelización (por contratar)

> Desarrollo de herramienta gráfica de administración

Componente Documental: w@rdA

> Desarrollo sobre solución comercial de IECISA (InvesDoc Corporate Server)

> Convenio de cesión de código fuente (posibilidad de cesión a otras AA.PP.)

$>$ Licencia de uso corporativa

$>100 \%$ tecnología java

> Integración nativa con Trew@

> Integración con @rchivA (Componente de Archivo)

Componente de archivo: @rchivA

$>$ Desarrollo a medida

> Código propiedad de la Junta de Andalucía

$>100 \%$ tecnología java

$>$ Gestión de archivos de gestión, centrales, intermedios e históricos

> Descripción de documentos basada en estándares (Moreq)

Buses de conexión

$>$ Desarrollo sobre plataforma BEA Web Logic Integrator

> Funcionalidad completa EAl (transporte, transformación, orquestación, etc)

$>100 \%$ tecnología java (JMS, JTA, JDBC, JCA...)

$>$ Nodo centralizado para toda la Junta de Andalucia (fiabilidad y escalabilidad garantizada) 